\title{
Carrier dynamics of electrons and holes in moderately doped silicon
}

\author{
Martin van Exter and D. Grischkowsky \\ IBM Research Division, Thomas J. Watson Research Center, P.O. Box 218, Yorktown Heights, New York 10598
}

(Received 22 December 1989)

\begin{abstract}
A time-domain spectroscopic technique, based on the generation and detection of a collimated beam of subpicosecond broadband terahertz pulses, is used to measure the absorption and dispersion of $n$ - and $p$-type silicon, with resistivities of $0.1,1$, and $10 \Omega \mathrm{cm}$ in the submillimeter range of 0.1-2 THz. From the transmission measurements performed at room temperature and at $80 \mathrm{~K}$, the absorption and dispersion, and concomitantly the full complex conductivity, of the doped silicon could be obtained. The results provide an accurate view on the dynamics of the electrons and the holes. Although the simple Drude model, with an energy-independent relaxation time, gives a surprisingly accurate description of the observed carrier dynamics, the measurements do show that some refinements are needed. An extended model, with an energy-dependent carrier-relaxation rate, can explain most of the observed deviations from the simple Drude model.
\end{abstract}

\section{INTRODUCTION}

For several decades physicists have studied the dynamics of carriers in semiconductors. A very interesting region for this study is the far-infrared or submillimeter region, where the frequency of the electromagnetic radiation is comparable to the carrier damping rate. ${ }^{1-7} \mathrm{Un}$ fortunately, this region is difficult to reach with available sources. In the microwave range below $100 \mathrm{GHz}$ semiconductor samples have been investigated through insertion in oversized waveguides. ${ }^{2}$ In these experiments it is important that the sample fits tightly in the waveguide, a situation that is never perfectly fulfilled, although the use of a circular $\mathrm{TE}_{01}$ mode reduces the problem dramatical$\mathrm{ly}^{2}$ A second complication is the variation in load experienced by the microwave source due to the reflections from the sample. Therefore, other groups have used horn-shaped transmitters and receivers to couple the microwave field into free space. ${ }^{6}$ However, microwave techniques in general have the disadvantage that they are done at one single frequency or at best over a narrow frequency band. With the well-developed technique of classical far-infrared spectroscopy the high-frequency (above $2 \mathrm{THz}$ ) behavior of semiconductors has been thoroughly investigated. ${ }^{1,4,7}$ However, for moderate doping, the strongest absorption of the free carriers lies below $2 \mathrm{THz}$, where classical far-infrared spectroscopy becomes difficult and time consuming, though some excellent studies have been performed. ${ }^{3,4}$ Consequently, the investigation of the most important frequency range from 0.1 to 2 $\mathrm{THz}$ has remained incomplete.

Recently a new system has become available for spectroscopic studies in the range from 0.1 to $2 \mathrm{THz}^{8-11}$ This system is based on the optoelectronic generation and reception of a beam of subpicosecond terahertz pulses. By inserting a sample in the beam and comparing the shape of the original subpicosecond terahertz pulses with the shapes of pulses that have propagated through the sample, one is able to deduce the frequency-dependent absorption and dispersion; this procedure is called time- domain spectroscopy.

In this paper we describe an application of timedomain spectroscopy with terahertz beams to a complete measurement of the absorption and dispersion due to carriers in doped silicon. The measured samples were $n$ - and $p$-doped silicon wafers, having a $\langle 111\rangle$ orientation and room-temperature resistivities of $0.1,1$, and $10 \Omega \mathrm{cm}$. The frequency-dependent properties are shown to be completely due to the carriers and not to the host crystal. Our measurements extend from 0.1 to $2 \mathrm{THz}$ and allow for the most comprehensive determination of the complex conductivity to date. To first order the results are reasonably well fit by the simple Drude theory for all the $n$-type and $p$-type samples and for both room temperature $(293 \mathrm{~K})$ and $80 \mathrm{~K}$. Slight but significant deviations indicate further refinements in the theory are needed. We show how a model that takes the frequency dependence of the carrier relaxation into account can explain practically all the observed deviations.

\section{THEORY}

The Drude model ${ }^{5-7}$ treats the free carriers in a solid as classical point charges subject to random collisions. In the first part of this paper the simplest version of the Drude model is adopted, in which the collision damping is independent of the carrier energy. The linear interaction between an isotropic medium and electromagnetic radiation is, in general, described by a frequencydependent complex dielectric constant $\epsilon$, being the square of the complex index of refraction $n=n_{r}+i n_{i}$. According to the Drude model the dielectric constant in SI units is

$$
\begin{aligned}
& \epsilon=\epsilon_{\infty}+\frac{i \sigma}{\omega \epsilon_{0}}=\epsilon_{\infty}-\frac{\omega_{p}^{2}}{\omega(\omega+i \Gamma)}, \\
& \sigma=\sigma_{\mathrm{dc}} \frac{i \Gamma}{\omega+i \Gamma}=\frac{i \epsilon_{0} \omega_{p}^{2}}{\omega+i \Gamma},
\end{aligned}
$$

where $\epsilon_{\infty}$ is the contribution of the dielectric, $\Gamma=1 / \tau$ is 
the damping rate, and $\tau$ is the average collision time. The plasma angular frequency $\omega_{p}$ is defined by $\omega_{p}^{2}=N e^{2} / \epsilon_{0} m$, where $N$ is the number density of carriers, $e$ is the electronic charge, $\epsilon_{0}$ is the free-space permittivity, and $m$ is the effective carrier mass. We have also presented the Drude result for the complex electric conductivity $\sigma$, where the dc conductivity $\sigma_{\mathrm{dc}}$ is given by $\sigma_{\mathrm{dc}}=e \mu N$, with the mobility $\mu$ given by $\mu=e / m \Gamma$. The electric field at the peak of the terahertz pulse is only of the order of $0.1 \mathrm{~V} / \mathrm{cm}$, corresponding to an average energy in the terahertz beam of about $10 \mathrm{nW} .{ }^{11}$ Clearly, this is small enough to validate the low-field expression for $\mu$ and to neglect joule heating. For the $80-\mathrm{K}$ samples and the highest frequencies, the energy of the far-infrared photons becomes almost $k T$. Dumke ${ }^{12}$ has shown that even for this case quantum effects give only a $1 \%$ correction compared to the classical model.

The effective carrier mass used in the Drude model represents the curvature of the energy-momentum relation of the carriers in silicon. Silicon has an indirect bandgap and the minimum of the conduction band is sixfold degenerate and close to the Brillouin zone in the $\langle 100\rangle$ direction. ${ }^{13}$ This asymmetry leads to the existence of a transverse and a longitudinal electron mass, $m_{t}$ and $m_{l}$, respectively. The effective electron mass $m_{e}$ is given by $\left(1 / m_{e}\right)=\left(2 / m_{t}+1 / m_{l}\right) / 3$. Using cyclotron resonance ${ }^{13,14}$ these masses have been measured to be $m_{t}=0.19 m_{0}$ and $m_{l}=0.92 m_{0}$, and thus $m_{e}=0.26 m_{0}$, where $m_{0}$ is the free-electron mass. The situation for the holes is more complicated, because there are three different valence bands around $k=0$ : there are light holes $\left(m_{\mathrm{lh}}=0.16 m_{0}\right)$ and heavy holes $\left(m_{\mathrm{hh}}=0.49 m_{0}\right)$ (Refs. 13 and 14) and there is an additional band split off due to spin-orbit interaction. The percentage of holes in the split-off band is about $2 \%$ at room temperature ${ }^{15}$ and drops quickly when the temperature is lowered. In this article, the influence of this band is neglected. For parabolic bands the ratio of heavy and light holes is easily calculated and the effective hole mass that enters the Drude model is $m_{h}=\left(m_{\mathrm{hh}}^{1.5}+m_{\mathrm{lh}}^{1.5}\right) /\left(m_{\mathrm{hh}}^{0.5}+m_{\mathrm{lh}}^{0.5}\right)$ and thus is $0.37 m_{0}$ (Refs. 1 and 13). This result is only valid to first order, as the heavy-hole band is nonparabolic and has a warped structure, which gives the effective hole mass a slight temperature dependence. ${ }^{15,16}$

Experimentally, we measure the amplitude transmission and the phase shift of terahertz radiation propagated through a flat sample. We convert the transmission into an intensity absorption coefficient $\alpha$, which is related to the above equations via $\alpha=n_{i}\left(4 \pi / \lambda_{0}\right)$. The measured phase shift is converted into the (real component of the) index of refraction, through division by the length of the sample. To link our optical measurements with highspeed electronics, we have also converted the experimental results into the complex high-frequency electrical conductivity $\sigma(\omega)$.

\section{EXPERIMENTAL TECHNIQUE}

The setup used to generate and detect the short pulses of terahertz radiation is depicted in Fig. 1 and has been described earlier. ${ }^{8-11}$ For the room-temperature experi-
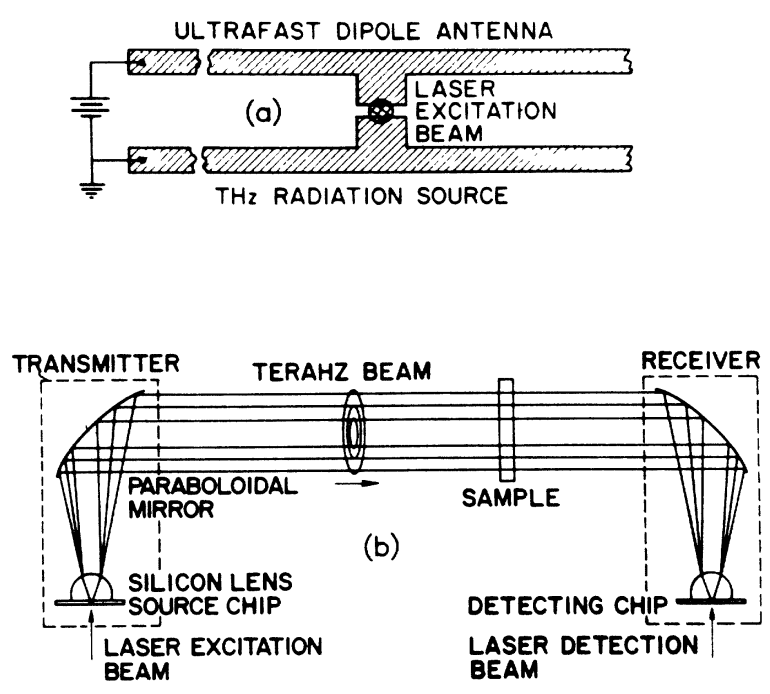

FIG. 1. (a) Antenna structure used in the experiment; (b) schematic for generation and detection of terahertz pulses.

ments we have used an identical transmitter and receiver. The dipole antenna, as shown in Fig. 1(a), consisted of two $20-\mu \mathrm{m}$-wide stubs separated by a $5-\mu \mathrm{m}$ gap, sticking inwards from two $10-\mu \mathrm{m}$ lines that were separated by 30 $\mu \mathrm{m}$. This structure was fabricated on an ion-implanted silicon-on-sapphire (SOS) wafer. The conductivity of the gap region is periodically changed through optoelectronic excitation by the $623 \mathrm{~nm}, 70 \mathrm{fs}$ pulses from a collidingpulse mode-locked (CPM) dye laser. The resulting current transients in the biased (10-V) transmitting antenna generate pulses of terahertz radiation. A large fraction of the generated terahertz radiation is captured by a silicon lens attached to the transmitting chip and is directed onto a paraboloidal mirror that recollimates the radiation into a low-divergence beam. This terahertz beam is directed towards the receiver, where it is focused onto the receiving antenna. The amplitude and time dependence of the transient voltage induced across the receiving antenna are obtained by measuring the collected charge (current) versus the time delay between the terahertz pulses and the laser pulses that synchronously gate the receiver. Such a measured transmitted terahertz pulse with no sample in place is shown in Fig. 2(a). The corresponding amplitude spectrum of this 0.5 -ps full width at half maximum (FWHM) pulse is presented in Fig. 2(c) and illustrates the $2-\mathrm{THz}$ bandwidth available for spectroscopy.

The available frequency range proved to be ideally suited for the study of moderately-doped silicon at room temperature. However, on cooling down to $80 \mathrm{~K}$ the carrier dynamics becomes considerably slower and the interesting spectral features shift to lower frequencies. The small and fast antennas we used at room temperature are not efficient enough at low frequencies, and our first measurements were very noisy. We therefore changed the receiver to a structure similar to that shown in Fig. 1(a), but with $120-\mu \mathrm{m}$ separation between the lines. The increased size of the antenna enhances the signal at low frequencies, where the detected spectral amplitude is pro- 

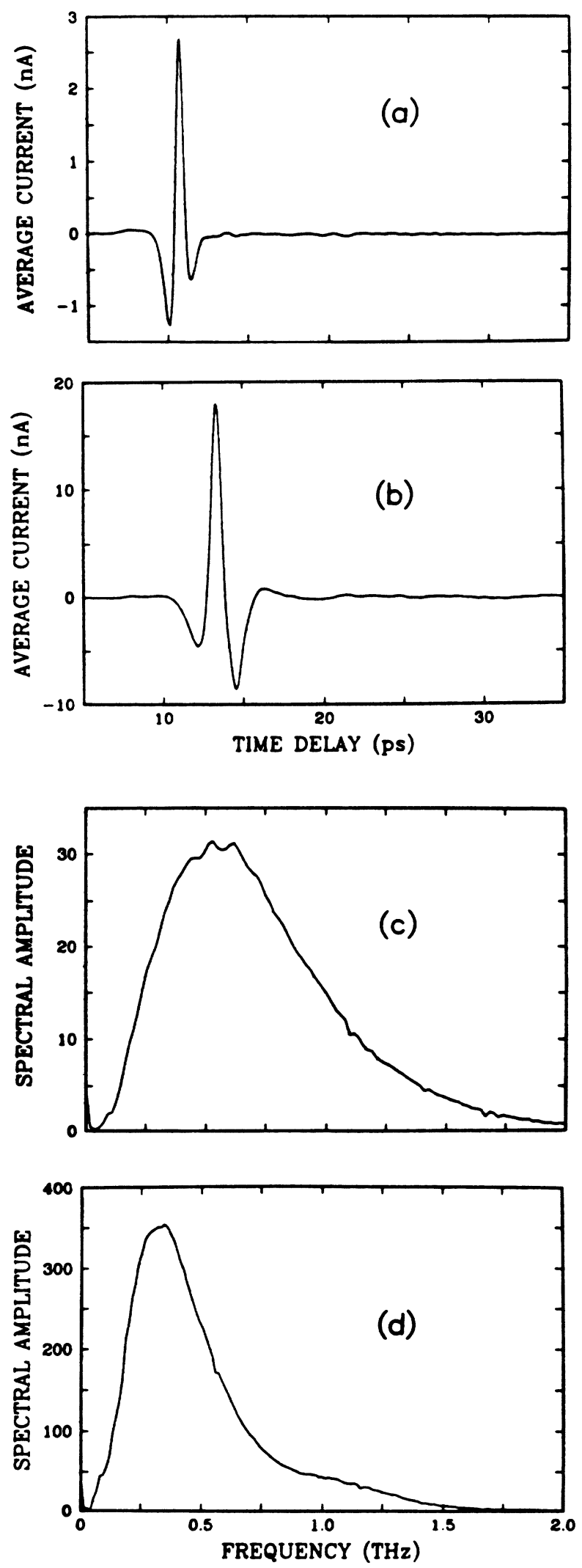

FIG. 2. (a) Terahertz pulse transmitted and detected with identical (10-30-10) antennas; (b) terahertz pulse generated with a (10-50-10) intrinsic GaAs transmitter and detected with a (10120-10) implanted silicon receiver; (c) amplitude spectrum of (a); (d) amplitude spectrum of (b). portional to the effective antenna length. The size of the transmitting antenna was increased to a (10-50-10) structure. The electromagnetic (EM) field radiated by a transient current is proportional to the time derivative of the current, in the far field and in the dipole approximation. Therefore, most terahertz radiation will be emitted during the fast rise time of the current transient and not during the slower fall time, being determined by the carrier lifetime of $0.6 \mathrm{ps}$ in implanted silicon. ${ }^{17}$ Experimentally, it proved to be advantageous to fabricate the transmitting antenna on an intrinsic GaAs wafer, instead of the usual ion-implanted SOS wafer. The use of GaAS increased the peak amplitude of the terahertz pulse by a factor of 5 , together with a slight increase in bandwidth. The terahertz pulse measured with this (10-50-10) GaAs transmitter and a (10-120-10) implanted silicon receiver is shown as Fig. 2(b) and the related spectrum is shown as Fig. 2(d). In Figs. 2(c) and 2(d) the same units are used, showing the advantage of long antennas below $0.5 \mathrm{THz}$. Unfortunately, the slower speed of the long antennas limits the usefulness of the (cold) high-frequency transmission data to 1.5 THz.

The terahertz beam, propagating from transmitter to receiver, has a complicated cross section, because the low-frequency components are more spread out than the high-frequency components. To remove the outer edges, the terahertz beam was passed through a $20-\mathrm{mm}$ diameter hole in a thin metal sheet positioned between the transmitter and the receiver. This reduced the peak signal by less than $10 \%$ and allowed us to use samples of moderate size. The room-temperature transmission measurements on doped silicon were performed by completely covering this circular hole by a flat wafer and monitoring the change in shape of the transmitted pulse. For the measurements at $80 \mathrm{~K}$, a small dewar with crystalline quartz windows was centered between the transmitter and receiver. The metal sample holder inside the dewar also had a 20 -mm-diameter opening. The reflection losses at the windows were $25 \%$ in total. The absorption of the two $\frac{3}{8}$-in.-thick crystalline quartz windows is small below $1 \mathrm{THz}$, but increases rapidly from about $15 \%$ at 1 $\mathrm{THz}$ to almost $50 \%$ at $1.5 \mathrm{THz}$. The dewar was a homemade liquid nitrogen container with a long cylindrical opening, in the middle of which the thin silicon wafer was mounted, using indium for thermal contact. The average equilibrium temperature of $80 \mathrm{~K}$ for our samples was measured for a typical cooling cycle of a thin silicon wafer, by a calibrated diode thermometer mounted at the center of the wafer.

In order to quantitatively explain the measurements on doped silicon, the index of refraction and absorption for undoped silicon was needed from 0.1 to $2.0 \mathrm{THz}$. Using time domain spectroscopy, we have measured these quantities with high precision for a $20-\mathrm{mm}$-thick piece of float-zone-refined, high-resistivity $(10 \mathrm{k} \Omega \mathrm{cm})$ silicon. The index was found to be 3.415 (Ref. 2) and essentially constant over the entire frequency range, being in good agreement with the literature value of 3.416 at $1.0 \mathrm{THz}{ }^{18}$ Using a thinner piece of silicon that fit in our dewar, we determined the index of refraction at $80 \mathrm{~K}$ to be $3.386 .^{5}$ This change is in reasonable agreement with the radio- 
frequency value of $\Delta n=-0.044$ (from 293 down to $77 \mathrm{~K}$ ) mentioned in (Ref. 19) and $\Delta n=0.00020 \mathrm{~K}^{-1}$ given in Ref. 20 (around $293 \mathrm{~K}$ at $25 \mathrm{~cm}^{-1}$ ). The high-resistivity silicon was almost transparent over the entire frequency range, with a measured intensity absorption of less than $0.04 \mathrm{~cm}^{-1}$.

\section{MEASUREMENT EXAMPLE}

We will now describe the results of transmission measurements on a $283-\mu$ m-thick, $25-\mathrm{mm}$-diameter wafer of $1.15 \Omega \mathrm{cm}, n$-type silicon at room temperature. This serves as an example of the possibilities and problems associated with the system and demonstrates the method of analysis used for all six wafers $(10,1$, and $0.1 \Omega \mathrm{cm}, n$, and $p$ type) at 293 as well as $80 \mathrm{~K}$. For the other samples we will only present the final results. The pulse sequence transmitted through the $1.15 \Omega \mathrm{cm}, n$-type silicon sample is shown in Fig. 3(a). The first transient is the primary pulse that passed directly through the sample. Its peak amplitude is about $40 \%$ of that of the original pulse. The output pulse amplitude and shape are changed due to reflection losses, and the frequency-dependent absorption and dispersion of the sample. The second and barelyresolvable third transients are terahertz pulses reflected inside the thin sample. Comparison of the Fourier transform of the single input pulse with that of the output pulse sequence from the doped silicon sample yields the frequency-dependent transmission, plotted as $-\ln \left[E_{\text {out }}(\omega) / E_{\text {in }}(\omega)\right]$ by the circles in Fig. 3(b). The multiple reflections are responsible for the oscillations in the frequency-dependent transmission of the wafer. Similar oscillations are also observed in the effective index of refraction obtained by division of the phase shift of the transmitted spectral components by the thickness of the wafer, which is plotted in Fig. 3(c) as circles. To include all these effects, we fit the measurements with a model for the transmission through a reflective, lossy parallel slab of material with frequency-dependent optical properties described by the Drude model. The solid lines in Figs. 3(b) and 3(c), are theoretical fits to the lossy slab model, using the two Drude parameters, the plasma angular frequency $\omega_{p}$ and the damping rate $\Gamma$. From the fit to the experimental data both $\omega_{p}$ and $\Gamma$ could be determined within $10 \%$ accuracy, using the eye to judge the fit quality.

To facilitate interpretation of the data, we have removed the influence of reflections numerically, using the dielectric constant obtained from the Drude fit. The results of the corrections are shown as dots in Figs. 3(b) and 3(c) and do not depend critically on the fit parameters. The corrections are especially important for the very high frequencies, where the absorption is low and the interference fringes are strong, and for the very low frequencies, where the index of refraction becomes large. Below $0.15 \mathrm{THz}$ the data becomes noisy due to the limited beam power, but the theoretically predicted minimum in the real part of the index of refraction followed by a dramatic rise towards low frequencies is clearly observable. Pure, high-resistivity silicon shows practically no absorption and hardly any frequency dependence of its index of refraction. Consequently, the observed strong frequency dependence for doped silicon is due to the carriers and not to the host crystal.

In Figs. 4(a) and 4(b) we have presented, respectively, the intensity absorption and the real part of the index of refraction for the discussed $1.15 \Omega \mathrm{cm}, n$-type silicon (dots) and for $0.92 \Omega \mathrm{cm}$, p-type silicon (circles) at $293 \mathrm{~K}$. As the terahertz optical properties of the samples are largely determined by the carrier dynamics, we have indirectly measured the electric conductivity of the doped silicon. To link our measurements with electronics, the real part of the electric conductivity calculated from the optical data is shown in Fig. 4(c) and the imaginary part is shown in Fig. 4(d). The real part of the conductivity is strongly frequency dependent, dropping monotonically from its dc peak to the reduced value at the highestmeasured frequency of $2 \mathrm{THz}$. In Table I the dc conduc-

TABLE I. Parameters used to fit the measurements in Figs. 4-8 with simple Drude theory. The columns indicate the wafer type $p$ or $n$, the resistivity $1 / \sigma_{\mathrm{dc}}(\Omega \mathrm{cm})$ as measured by the four-point contact method at room temperature, the temperature $T$, the low-frequency resistivity $1 / \sigma_{\mathrm{dc}}(\Omega \mathrm{cm})$ as extrapolated from the fitted curves, the measured plasma frequency $\omega_{p} / 2 \pi(\mathrm{THz})$, the measured damping rate $\Gamma / 2 \pi(\mathrm{THz})$, and the resulting mobility $\mu(\mathrm{cm} / \mathrm{V} \mathrm{s})$ determined by $\Gamma$, and carrier density $N\left(\mathrm{~cm}^{-3}\right)$ determined by $\omega_{p}$.

\begin{tabular}{llllllll}
\hline \hline Type & $1 / \sigma_{\mathrm{dc}}$ & \multicolumn{1}{c}{$T$} & $1 / \sigma_{\mathrm{dc}}$ & $\omega_{p} / 2 \pi$ & $\Gamma / 2 \pi$ & $\mu$ & $N$ \\
\hline$p$ & 9.0 & $293 \mathrm{~K}$ & 9.3 & 0.48 & 1.19 & 640 & $1.1 \times 10^{15}$ \\
$p$ & 0.92 & $293 \mathrm{~K}$ & 0.89 & 1.75 & 1.51 & 500 & $1.4 \times 10^{16}$ \\
$p$ & 0.164 & $293 \mathrm{~K}$ & & & & & \\
$n$ & 8.1 & $293 \mathrm{~K}$ & 8.2 & 0.36 & 0.59 & 1820 & $4.2 \times 10^{14}$ \\
$n$ & 1.15 & $293 \mathrm{~K}$ & 1.13 & 1.01 & 0.64 & 1680 & $3.3 \times 10^{15}$ \\
$n$ & 0.129 & $293 \mathrm{~K}$ & & & & & \\
$p$ & 9.0 & $80 \mathrm{~K}$ & 1.67 & 0.37 & 0.127 & 5960 & $6.3 \times 10^{14}$ \\
$p$ & 0.92 & $80 \mathrm{~K}$ & 0.42 & 0.92 & 0.198 & 3820 & $3.9 \times 10^{15}$ \\
$p$ & 0.164 & $80 \mathrm{~K}$ & 0.294 & 1.80 & 0.53 & 1430 & $1.5 \times 10^{16}$ \\
$n$ & 8.1 & $80 \mathrm{~K}$ & 1.45 & 0.33 & 0.088 & 12200 & $3.5 \times 10^{14}$ \\
$n$ & 1.15 & $80 \mathrm{~K}$ & 0.32 & 0.78 & 0.107 & 10100 & $2.0 \times 10^{15}$ \\
$n$ & 0.129 & $80 \mathrm{~K}$ & 0.180 & 2.00 & 0.40 & 2690 & $1.3 \times 10^{16}$ \\
\hline \hline
\end{tabular}


tivity extrapolated from the optical results is compared with a direct measurement at room temperature using the four-point contact method. ${ }^{21}$ This independent check on the derived Drude parameters shows excellent agreement. The behavior of the imaginary part of the conductivity is completely different, increasing from zero at low frequencies, peaking at midrange and then showing a gradual decline. The calculation of the imaginary part of the conductivity depends critically on the thickness of the sample. For that reason the calculated value might be as
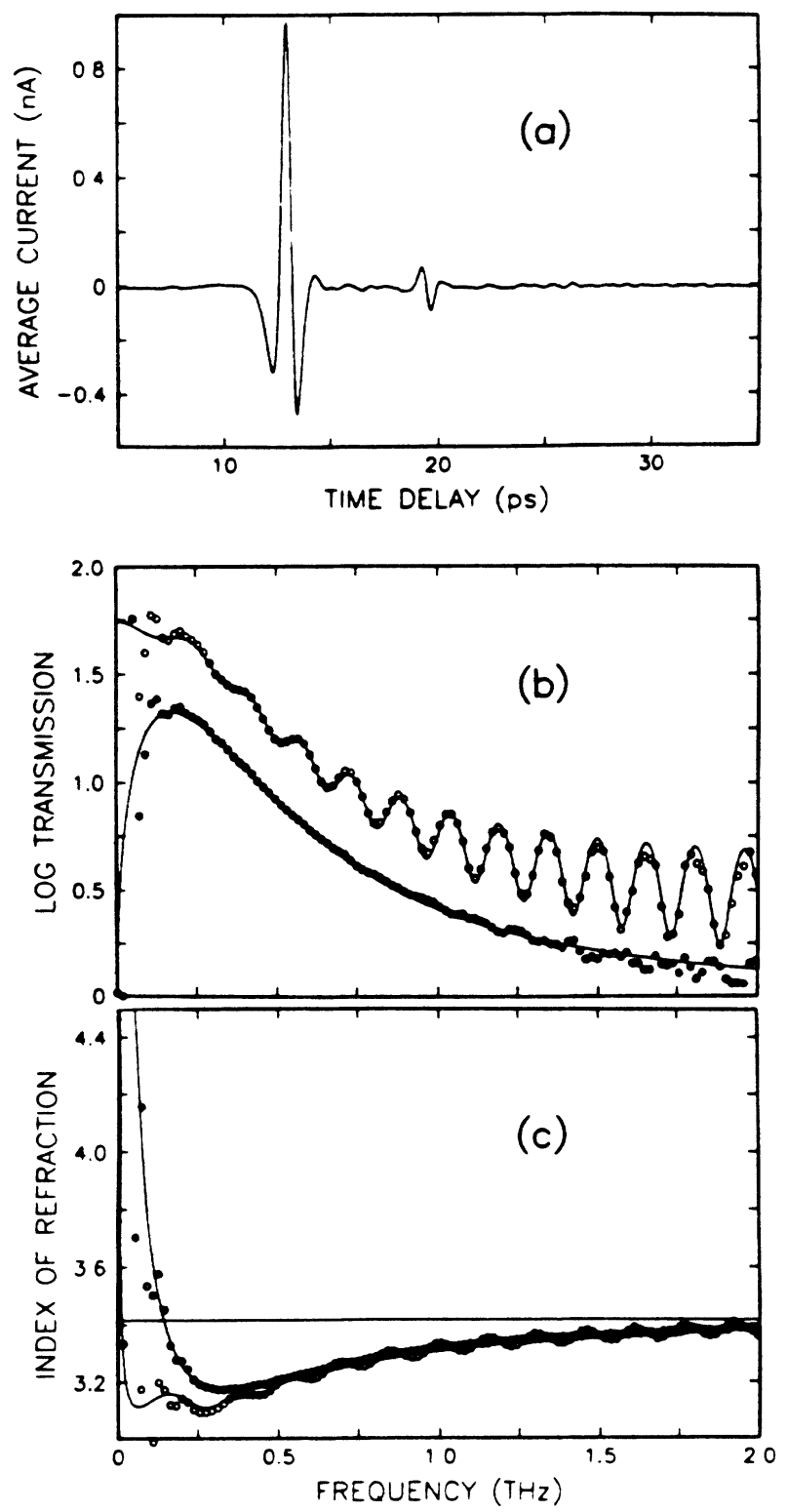

FIG. 3. (a) Terahertz pulse transmitted through a $283-\mu \mathrm{m}$ thick wafer of $1.15 \Omega \mathrm{cm}, n$-type silicon at room temperature; (b) logarithm of the amplitude transmission for this sample (circles) and the amplitude transmission numerically corrected for reflections (dots); (c) effective index of refraction, derived by division of the measured phase shift of the transmitted terahertz pulses by the length of the sample (circles) and the index of refraction corrected for reflections (dots).
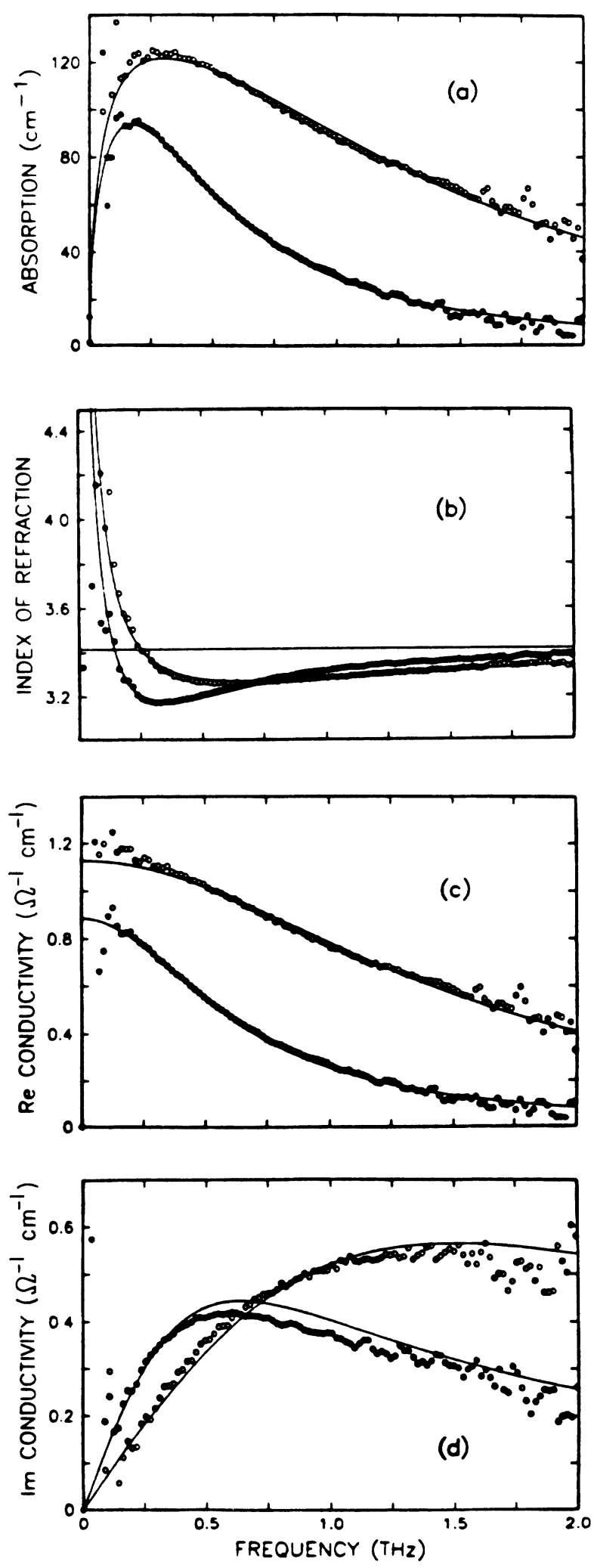

FIG. 4. Results for $1.15 \Omega \mathrm{cm}, n$-type (dots) and $0.92 \Omega \mathrm{cm}$, p-type (circles) silicon at room temperature. Solid lines describe simple Drude theory with parameters from Table I. (a) Intensity absorption; (b) real part of the index of refraction; (c) real part of the electric conductivity; (d) imaginary part of the electric conductivity. 
much as $10 \%$ off from the actual value. Keeping this in mind, the agreement with the Drude theory of both the real and imaginary part of the conductivity is quite acceptable.

\section{RESULTS}

The measurements of $1 \Omega \mathrm{cm}, p$ - and $n$-type silicon immediately show the different dynamic behavior of the electrons and the holes. At room temperature the damping rate $\Gamma$ in these moderately doped samples is mainly determined by carrier-phonon collisions, ${ }^{2,4,22}$ a process that apparently is more efficient for holes than for electrons. The results for a $1.459-\mathrm{mm}$-thick disc of $8.1 \Omega \mathrm{cm}$, $n$-type silicon and a $1.479-\mathrm{mm}$-thick disc of $9.0 \Omega \mathrm{cm}, p$ type silicon are shown as Fig. 5 and are also presented in Table I. The damping rate in these samples was slightly less than in the $1 \Omega \mathrm{cm}$ samples. At room temperature the $260-\mu \mathrm{m}$-thick, $0.1 \Omega \mathrm{cm}$ silicon samples we investigated were practically opaque and showed an amplitude transmission of less than $0.1 \%$. The transmitted pulses were too weak to analyze properly, and for room temperature the $0.1 \Omega \mathrm{cm}$ columns in Table $I$ unfortunately remain blank.

Combining the measured damping rates with the effective carrier masses, yields mobilities of 1680 $\mathrm{cm}^{2} \mathrm{~V}^{-1} \mathrm{~s}^{-1}$ for the electrons in the $1.15 \Omega \mathrm{cm}$ sample and $1820 \mathrm{~cm}^{2} \mathrm{~V}^{-1} \mathrm{~s}^{-1}$ for the $8.05 \Omega \mathrm{cm}$ sample. These values are higher than the literature value, which is 1450 $\mathrm{cm}^{2} \mathrm{~V}^{-1} \mathrm{~s}^{-1}$ for intrinsic silicon and decreases monotonically as the doping level increases. ${ }^{13,23,24}$ Usually the carrier mobility is determined either directly in a time-offlight experiment, ${ }^{23}$ or via the Hall effect. Hall measurements should be used with care, because they determine the so-called Hall mobility, which differs from the (electric) mobility by the Hall factor. This factor depends on the ratio of longitudinal over transverse carrier mass, as well as on the model-dependent factor $\left\langle\tau^{2}\right\rangle /\langle\tau\rangle^{2}$, where \langle\rangle signifies an average over the carrier distribution. ${ }^{24,25}$ For the hole mobility we find $500 \mathrm{~cm}^{2} \mathrm{~V}^{-1} \mathrm{~s}^{-1}$ for the $0.92 \Omega \mathrm{cm}$ sample and $640 \mathrm{~cm}^{2} \mathrm{~V}^{-1} \mathrm{~s}^{-1}$ for the $9.0 \Omega \mathrm{cm}$ sample. These values are also higher than the literature value of $450 \mathrm{~cm}^{2} \mathrm{~V}^{-1} \mathrm{~s}^{-1}$ for intrinsic silicon. ${ }^{13}$ If we stick to the simple Drude model we are facing a clear disagreement between the damping rate extracted from the transmission experiments and the mobility extracted from other experiments. This discrepancy supports an energy dependence of the carrier collision time as will be discussed at the end of this paper.

The carrier density of the various samples can be easily calculated from the measured plasma frequency with the use of the literature value for the effective mass. The difference in plasma frequency between $p$ - and $n$-type samples mainly reflects the larger doping needed in the $p$-type to compensate for the higher damping rate and get the same dc conductivity. The carrier densities we calculate are presented in Table I. They are rather low compared to the doping levels of $1.4 \times 10^{15}, 1.8 \times 10^{16}$, and $2.9 \times 10^{17} \mathrm{~cm}^{-3}$ for the 10,1 , and $0.1 \Omega \mathrm{cm}, p$-type silicon, respectively, and $7.0 \times 10^{14}, 4.6 \times 10^{15}$, and $6.6 \times 10^{16} \mathrm{~cm}^{-3}$ for the 10,1 , and $0.1 \Omega \mathrm{cm}, n$-type sil-
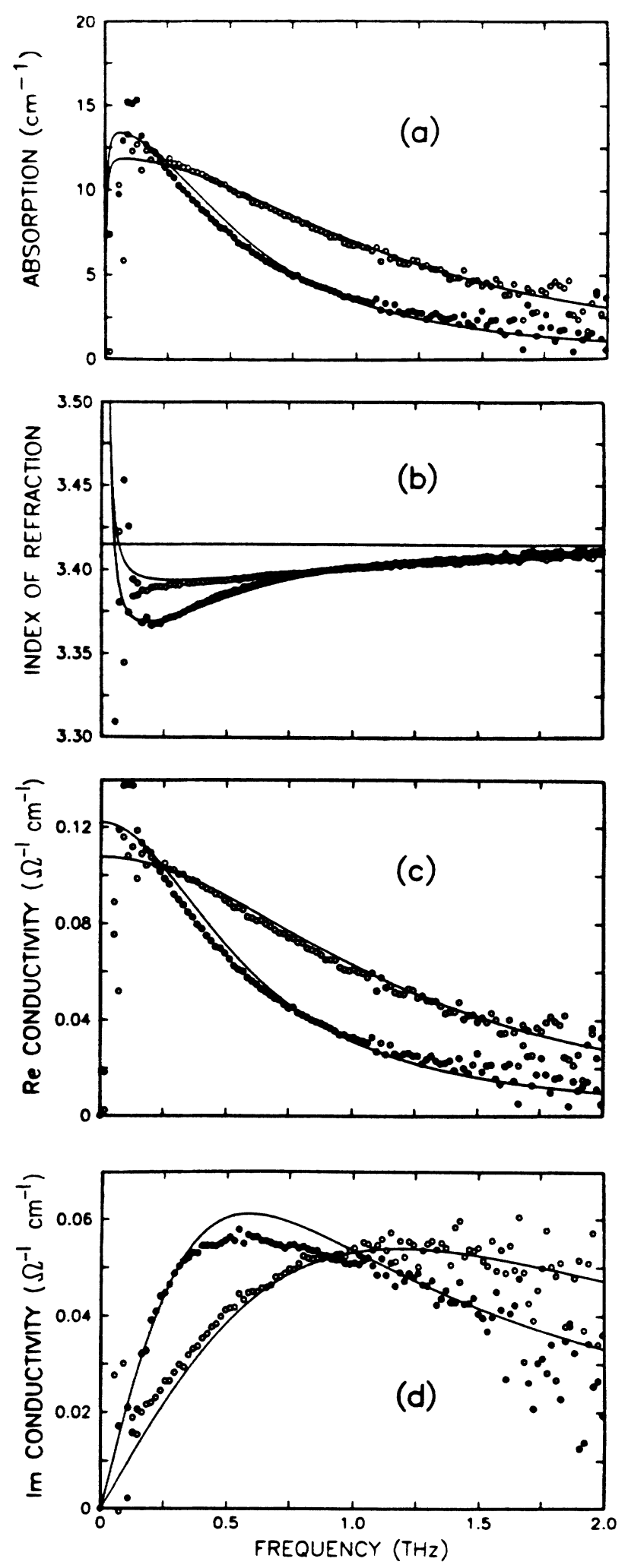

FIG. 5. Results for $8.1 \Omega \mathrm{cm}, n$-type (dots) and $9.0 \Omega \mathrm{cm}, p$ type (circles) silicon at room temperature. Solid lines describe simple Drude theory with parameters from Table I. (a) Intensity absorption; (b) real part of the index of refraction; (c) real part of the electric conductivity; (d) imaginary part of the electric conductivity. 
icon, given by the manufacturer (Virginia semiconductor) with an error margin of $20 \%$.

It is interesting to point out that $\sigma_{\mathrm{dc}}$, calculated from the relationship $\sigma_{\mathrm{dc}}=e \mu N$ with $\mu$ and $N$ obtained from Table I, agrees exceptionally well with both $\sigma_{\mathrm{dc}}$ obtained by extrapolating the $\operatorname{Re} \sigma(\omega)$ curve and $\sigma_{\mathrm{dc}}$ as measured by the four-probe method. Thus, with respect to the above comments concerning $\mu$ and $N$, it appears that the simple Drude model overestimates $\mu$, but that it underestimates $N$ by the same percentage. The end result is that $\sigma_{\mathrm{dc}}$, is given accurately.

Figures 6-8 show the results of terahertz transmission measurements on the previously mentioned doped silicon samples at the cryogenic temperature of $80 \mathrm{~K}$. At this temperature the average amplitude transmission of the $0.129 \Omega \mathrm{cm}, n$ type and the $0.164 \Omega \mathrm{cm}, p$-type sample has risen to about $4 \%$ and can now be easily analyzed. Upon cooling, the spectral features of all the samples have shifted to lower frequencies and the frequency dependence has become even more pronounced. The peaks have increased and sharpened and the minima have deepened and narrowed. At the highest measured frequency of 1.5 $\mathrm{THz}$, the optical effects due to the carriers are monotonically disappearing in the 10 and $1 \Omega \mathrm{cm}$ samples. Upon cooling the dc conductivity of the 10 and $1 \Omega \mathrm{cm}$ samples have increased, while the falloff towards increasing frequency has steepened. However, for the $0.1 \Omega \mathrm{cm}$ samples, due to their proportionally more severe carrier trapping at the dopant, the conductivities have significantly decreased. The Drude parameters derived from these measurements are presented in Table I. The slower carrier dynamics is reflected in the strong reduction in damping rate $\Gamma$. The decrease in plasma frequency signifies a reduction in carrier density $N$ related to carrier trapping at the dopant. For all the samples the measured decrease in $N$ agrees well with that calculated due to the change in temperature and the corresponding shift of the Fermi level, using a value of $45 \mathrm{meV}$ for the ionization energy of both the phosphorus dopant used in $n$-type silicon and the boron used in $p$-type silicon. ${ }^{13}$

The quality of the Drude fit for the cold samples is not as precise as for the room temperature samples. The deviations are best observed for the $p$-type material. For the $10 \Omega \mathrm{cm}$ silicon the index of refraction dropped more than expected and did not show the usual increase towards low frequencies [Fig. 6(b)], perhaps due to the large increase in noise below $0.1 \mathrm{THz}$. For the $1 \Omega \mathrm{cm}$, p-type silicon (Fig. 7), the measured absorption at high frequencies is larger than predicted by the Drude model, while it is slightly less at lower frequencies. Additionally, the measured minimum in the index of refraction, plotted in Fig. 7(b), comes at a lower frequency than expected from the Drude model. For the more heavily doped 0.1 $\Omega \mathrm{cm}$ silicon the same deviations from the Drude model show up, but are more severe (Fig. 8). The deviations between the theoretical curves and the measurements could not be removed through variation of the two fit parameters, which should also fit both the absorption and the index of refraction data. The deviations are reproducible from run to run and thus signify a disagreement with the simple Drude model for the cold samples. This
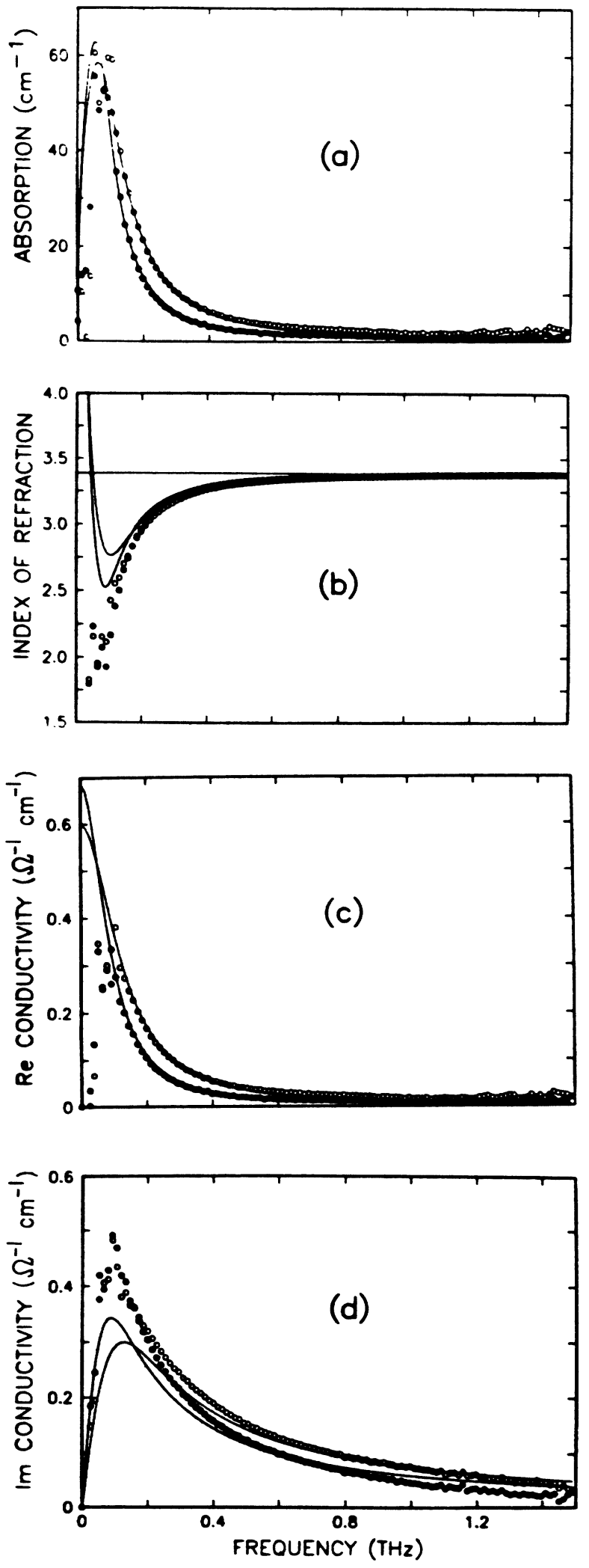

FIG. 6. Results for $8.1 \Omega \mathrm{cm}, n$-type (dots) and $9.0 \Omega \mathrm{cm}, p$ type (circles) silicon at $80 \mathrm{~K}$. Solid lines describe simple Drude theory with parameters from Table I. (a) Intensity absorption; (b) real part of the index of refraction; (c) real part of the electric conductivity; (d) imaginary part of the electric conductivity. 

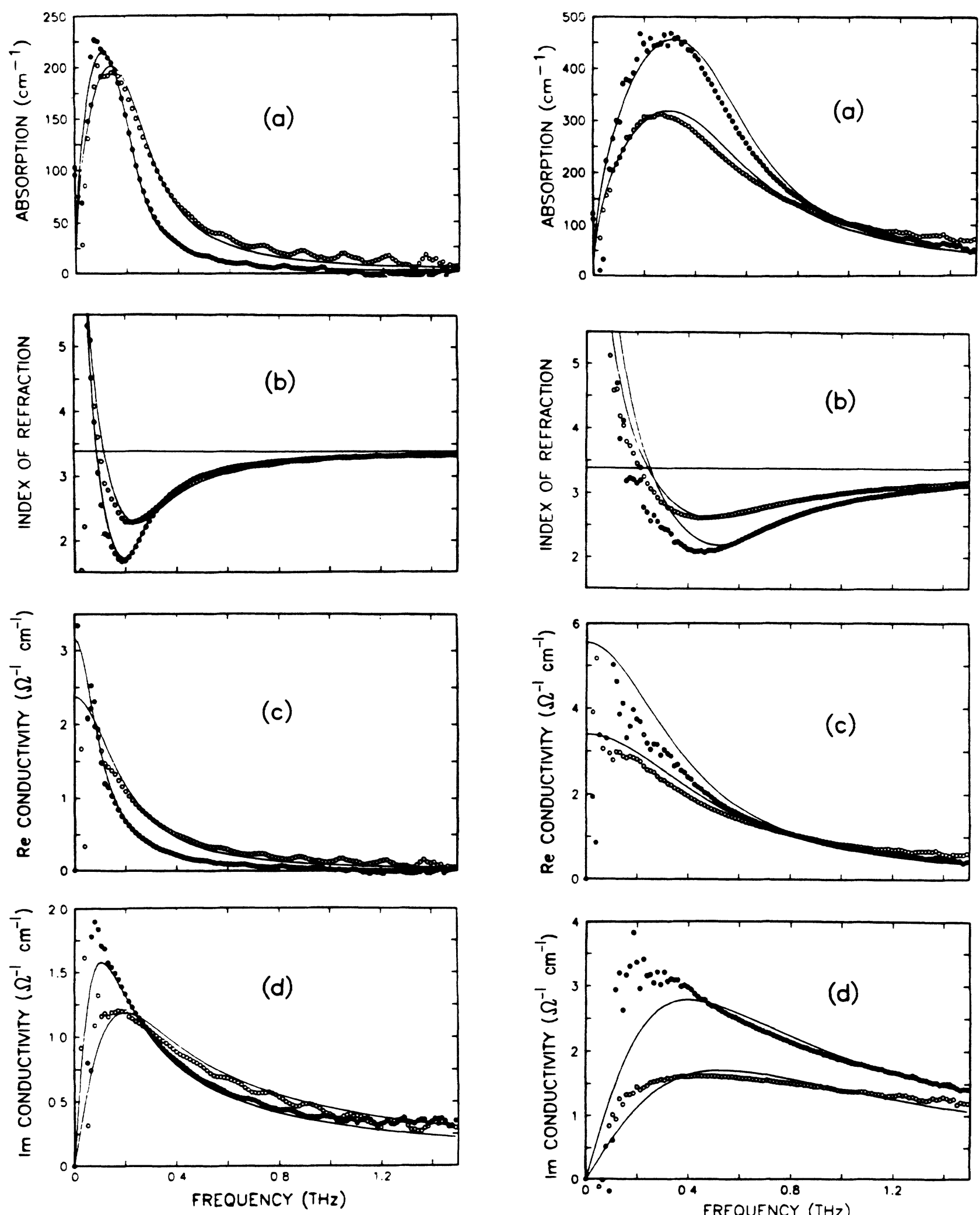

FIG. 7. Results for $1.15 \Omega \mathrm{cm}, n$-type (dots) and $0.92 \Omega \mathrm{cm}$, p-type (circles) silicon at $80 \mathrm{~K}$. Solid lines describe simple Drude theory with parameters from Table I. (a) Intensity absorption; (b) real part of the index of refraction; (c) real part of the electric conductivity; (d) imaginary part of the electric conductivity.

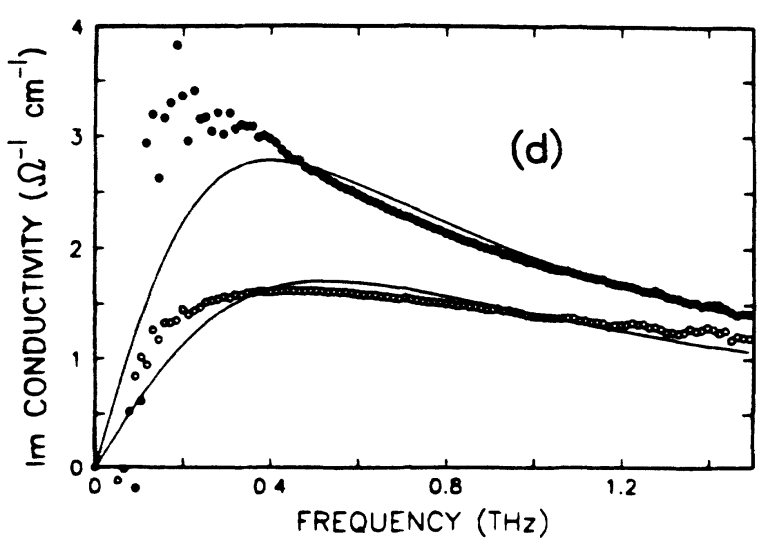

FIG. 8. Results for $0.129 \Omega \mathrm{cm}, n$-type (dots) and $0.164 \Omega \mathrm{cm}$, p-type (circles) silicon at $80 \mathrm{~K}$. Solid lines describe simple Drude theory with parameters from Table I. (a) Intensity absorption; (b) real part of the index of refraction; (c) real part of the electric conductivity; (d) imaginary part of the electric conductivity. 
discrepancy is considered to be due to an energy dependence of the collision time. In the following section we will briefly discuss an extended model for the carrier dynamics, which distinguishes between scattering with phonons and scattering with ionized impurities. A complication we will not take into account is the existence of both light holes and heavy holes and holes in the splitoff valence band, all of which may display a different dynamic behavior.

\section{EXTENDED MODEL AND CONCLUSIONS}

Many sophisticated models have been developed to give a more accurate description of the carrier dynamics that goes beyond the simple Drude model. ${ }^{2,4,25-31}$ In these models the carrier relaxation is split into both intervalley and intravalley phonon scattering and scattering with the ionized impurities. Additionally, the interaction with the lattice is split into scattering from acoustic phonons and optic phonons. The many parameters involved make these extended models complicated to work with. Consequently, as a first-order approximation, we have analyzed our data with the more transparent simple Drude model.

We will now discuss the qualitative changes resulting from the energy dependence of the carrier relaxation. Theory predicts ${ }^{27,28,32}$ that the efficiency of electronphonon collision increases with the carrier energy to the power 0.5 , because the mean free path for electronphonon interaction is independent of carrier energy. In contrast, the carrier scattering from ionized impurities is more efficient at lower energies, scales roughly as the carrier energy to the power -1.5 , and will dominate at low temperatures. $^{27,28,32}$ The damping rate of carriers with energy $E$ relative to the extreme of the band is thus given by

$$
\Gamma(E)=\Gamma_{l}\left(E / k T_{0}\right)^{0.5}+\Gamma_{i}\left(E / k T_{0}\right)^{-1.5},
$$

where the coupling constants $\Gamma_{l}$ and $\Gamma_{i}$ characterize the efficiency of scattering with the lattice and the ionized impurities, respectively, and depend on temperature and doping. $T_{0}$ is a normalization parameter, with the dimension temperature. In this way both $\Gamma_{l}$ and $\Gamma_{i}$ have the same dimension as the energy-independent damping rate used in the simple Drude model. The optical and electric properties of the doped semiconductor can now be evaluated by using the Drude result for each carrier energy and integrating over the conduction (or valence) band, as shown by the three examples in Figs. 9(a)-9(d). Using the Boltzmann transport equation one can show that, $5,28,29,32$

$$
\sigma=\sigma_{\mathrm{dc}}\left\langle\frac{i \Gamma(E)}{\omega+i \Gamma(E)}\right\rangle=i \epsilon_{0} \omega_{p}^{2}\left\langle\frac{1}{\omega+i \Gamma(E)}\right\rangle,
$$

where \langle\rangle denotes an average weighted by the product of the distribution of the carriers times their energy. For a parabolic band the weight factor is $E^{1.5} e^{-(E / k T)}$. The dc conductivity is given by $\sigma_{\mathrm{dc}}=\left(e^{2} N / m\right)\langle 1 / \Gamma\rangle$ and the relation between $\sigma$ and $\epsilon$ remains unchanged.

Inserting the energy-dependent damping rate into the equation for the frequency-dependent conductivity, one
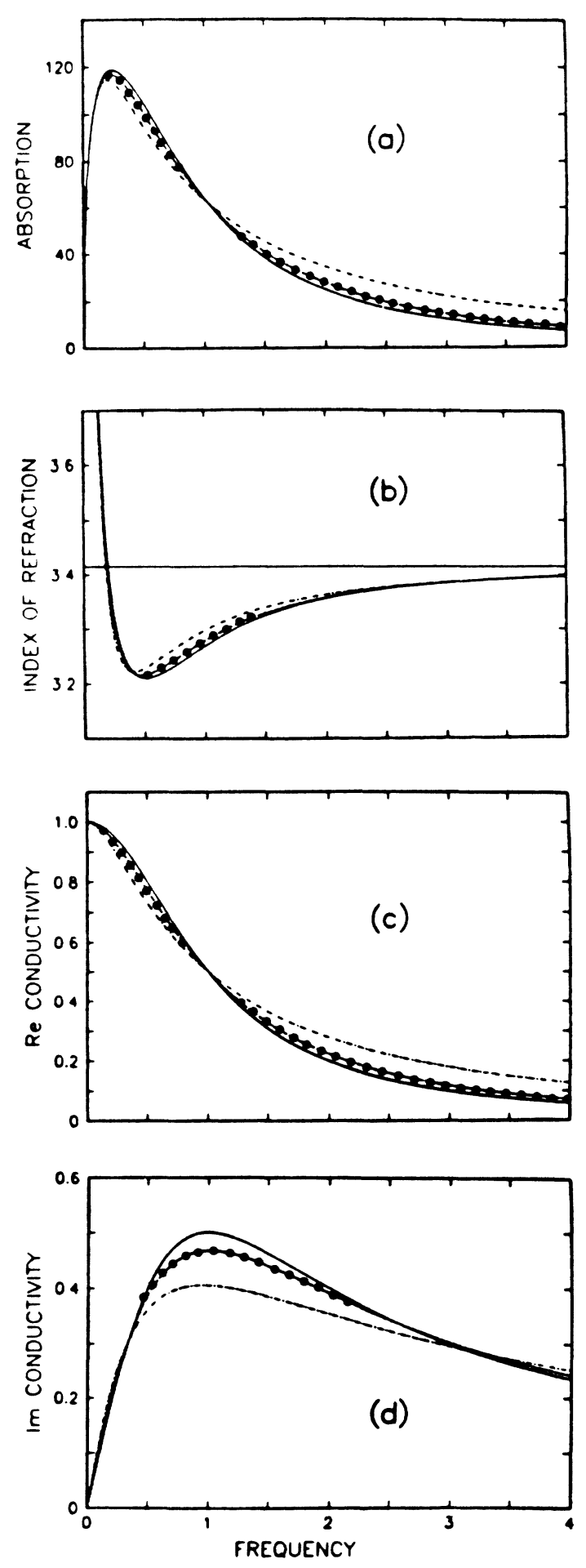

FIG. 9. Calculated optical and electronic properties for three different models for the carrier dynamics. The simple Drude model with an energy-independent damping rate is indicated by the solid line. The dotted curve describes phonon scattering only, with $\Gamma(E)$ proportional to $E^{0.5}$. The dashed curve describes scattering with impurities, with $\Gamma(E)$ proportional to $E^{-1.5}$. The coupling constants and carrier densities are adjusted to give identical dc conductivities and similar half-widths for the curves of $\operatorname{Re} \sigma(\omega)$; (a) intensity absorption; (b) real part of the index of refraction; (c) real part of the electric conductivity; (d) imaginary part of the electric conductivity. 
can evaluate certain universal curves $^{4}$ for, e.g., the $\operatorname{Re} \sigma(\omega)$ versus $\omega$ relation. The scaling behavior of this function is simple, and it is easily adjusted to a specific case. A change in carrier density, e.g., only affects the conductivity axis, but leaves the frequency dependence untouched. If both coupling constants are increased by the same factor, the $\operatorname{Re} \sigma(\omega)$ versus $\omega$ curve flattens out, but retains its specific shape. The three curves in Fig. 9(c) show the calculated real part of the conductivity for the simple Drude model, for phonon scattering only $\left(\Gamma_{l}=0.8, \Gamma_{i}=0\right)$ and for impurity scattering only $\left(\Gamma_{l}=0, \Gamma_{i}=6.8\right)$. Both axes are in arbitrary units, but can be easily adjusted to a specific case as explained above. The coupling constants $\Gamma_{l}$ and $\Gamma_{l}$ were adjusted to give the same half-width for the three curves. The area under the $\operatorname{Re} \sigma(\omega)$ versus $\omega$ curve is directly proportional to the carrier density and independent of the functional dependence of the carrier damping rate. It is interesting to note that we had to increase the carrier density by $6 \%$ for the pure phonon-scattering process and by as much as $51 \%$ for the pure impurity scattering case to obtain the same dc conductivity as in the simple Drude model. This shows that when real data, which presumably includes both phonon and impurity scattering, is fit with a simple Drude model, using the dc conductivity and the half-width as fit criteria, one will always underes- timate the carrier density and thereby overestimate the mobility. This is in agreement with our results for the room-temperature samples. In the Figs. 9(a), 9(b), and 9(d), we have plotted the other terahertz properties for the same three cases. The deviations from the simple Drude model are most dramatic for pure impurity scattering and are qualitatively similar to the deviations observed in the experimental data, being most dramatic for the $80-\mathrm{K} 0.1 \Omega \mathrm{cm}$ silicon. The absorption is too small at low frequencies and too large at high frequencies, and the minimum in the index of refraction comes at lower frequencies than expected from the Drude theory, while it is slightly high in the midfrequency range. A careful inspection of the room-temperature data shows similar, but much smaller, deviations. This gives us confidence that time-resolved spectroscopy in the frequency range from 0.1 to $2 \mathrm{THz}$ is a very sensitive method to study the dynamics of carriers in semiconductors, allowing one to separate and individually observe their various relaxation channels.

\section{ACKNOWLEDGMENTS}

We acknowledge informative discussions with Søren Keiding and the excellent masks and wafer fabrication by Hoi Chan. This research was partially supported by the U.S. Office of Naval Research.
${ }^{1}$ W. G. Spitzer and H. Y. Fan, Phys. Rev. 106, 882 (1957).

${ }^{2}$ J. D. Holm and K. S. Champlin, J. Appl. Phys. 39, 275 (1968).

${ }^{3}$ S. J. Allen, Jr., D. C. Tsui, and F. DeRosa, Phys. Rev. Lett. 35, 1359 (1975).

${ }^{4} \mathrm{M}$. Vindevoghel, J. Vindevoghel, and Y. Leroy, Infrared Phys. 15, 161 (1975).

${ }^{5}$ N. W. Ashcroft and N. D. Mermin, Solid State Physics (Holt, Rinehart, and Winston, New York, 1976).

${ }^{6}$ R. T. Kinasewitz and B. Senitzky, J. Appl. Phys. 54, 3394 (1983).

${ }^{7}$ T. Ohba and S. Ikawa, J. Appl. Phys. 64, 4141 (1988).

${ }^{8}$ Ch. Fattinger and D. Grischkowsky, Appl. Phys. Lett. 53, 1480 (1988); 54, 490 (1989).

${ }^{9}$ M. van Exter, Ch. Fattinger, and D. Grischkowsky, Appl. Phys. Lett. 55, 337 (1989).

${ }^{10} \mathrm{M}$. van Exter, Ch. Fattinger, and D. Grischkowsky, Opt. Lett. 14, 1128 (1989).

${ }^{11}$ M. van Exter and D. Grischkowsky, IEEE Trans. Microwave Theory Tech. (to be published).

${ }^{12}$ W. P. Dumke, Phys. Rev. 124, 1813 (1961).

${ }^{13}$ S. M. Sze, Physics of Semiconductor Devices (Wiley, New York, 1981).

${ }^{14}$ R. N. Dexter, H. J. Zeiger, and B. Lax, Phys. Rev. 104, 637 (1956).

${ }^{15}$ G. Ottaviani, L. Reggiani, C. Canali, F. Nava, and A. Albergi-Quaranta, Phys. Rev. B 12, 3318 (1975).

${ }^{16}$ H. Miyazawa, K. Suzuki, and H. Maeda, Phys. Rev. 131, 2442 (1963).
${ }^{17}$ F. E. Doany, D. Grischkowsky, and C. C. Chi, Appl. Phys. Lett. 50, 460 (1987).

${ }^{18}$ E. V. Loewenstein, D. R. Smith, and R. L. Morgan, Appl. Opt. 12, 398 (1973).

${ }^{19}$ M. Cardona, W. Paul, and H. Brooks, in Solid State Physics in Electronics and Telecommunications, Proceedings of the International Conference on Solid State Physics in Electronics and Communications, Brussels, 1958, edited by M. Desirant and J. L. Michiels (Academic, London, 1960), Vol. 1, p. 206.

${ }^{20}$ J. R. Birch, Infrared Phys. 18, 613 (1978).

${ }^{21}$ L. B. Valdes, Proc. IRE 42, 420 (1954).

${ }^{22}$ C. Jacoboni, C. Canali, G. Ottaviani, and A. AlberigiQuaranta, Solid State Electron. 20, 77 (1977).

${ }^{23}$ M. B. Prince, Phys. Rev. 92, 681 (1953), 93, 1204 (1954).

${ }^{24}$ F. Mousty, P. Ostoja, and L. Passari, J. Appl. Phys. 45, 4576 (1974).

${ }^{25}$ C. Herring and E. Vogt, Phys. Rev. 101, 944 (1956).

${ }^{26}$ D. Long and J. Myers, Phys. Rev. 120, 39 (1960).

${ }^{27}$ D. Long, Phys. Rev. 120, 2024 (1960).

${ }^{28}$ J. M. Ziman, Electrons and Phonons (Oxford University Press, London, 1960).

${ }^{29}$ P. A. Schumann, Jr. and R. P. Phillips, Solid State Electron. 10, 943 (1967).

${ }^{30}$ P. K. Basu and B. R. Nag, Phys. Status Solidi B 53, K61 (1972).

${ }^{31}$ E. Barta, Infrared Phys. 17, 111 (1977).

${ }^{32}$ R. A. Smith, Semiconductors (Cambridge University Press, London, 1959). 